\title{
Modeling, Control and Design Optimization for a Fully-actuated Hexarotor Aerial Vehicle with Tilted Propellers
}

\author{
Sujit Rajappa ${ }^{1}$, Markus Ryll ${ }^{3}$, Heinrich H. Bülthoff ${ }^{1,2}$ and Antonio Franchi ${ }^{3,4}$
}

\begin{abstract}
Mobility of a hexarotor UAV in its standard configuration is limited, since all the propeller force vectors are parallel and they achieve only 4-DoF actuation, similar, e.g., to quadrotors. As a consequence, the hexarotor pose cannot track an arbitrary trajectory while the center of mass is tracking a position trajectory. In this paper, we consider a different hexarotor architecture where propellers are tilted, without the need of any additional hardware. In this way, the hexarotor gains a 6-DoF actuation which allows to independently reach positions and orientations in free space and to be able to exert forces on the environment to resist any wrench for aerial manipulation tasks. After deriving the dynamical model of the proposed hexarotor, we discuss the controllability and the tilt angle optimization to reduce the control effort for the specific task. An exact feedback linearization and decoupling control law is proposed based on the input-output mapping, considering the Jacobian and task acceleration, for non-linear trajectory tracking. The capabilities of our approach are shown by simulation results.
\end{abstract}

\section{INTRODUCTION}

Research in the field and applications related to unmanned aerial vehicles (UAVs) has been very popular in recent times, see e.g., [1] and references therein. The application possibility to use the UAV for various tasks such as search and rescue operation, exploration, surveillance, cooperative swarm tasks or transportation are all increasing and has been the main research subject with growing interest in the last decade with many industrial collaborations. Lately, the mobile manipulation tasks by aerial vehicles and physical interaction with the environment for various applications has been growing ground within the UAV community. The interaction can be done by direct contact [2]-[5], by considering simple grasping/manipulation tasks [6], [7] and has moved forward to multiple collaborative interactive UAVs [8].

Among the many challenges faced by typical UAVs, such as little flight time, limited payload capacity, uncertainties in outside environment etc., an important one is the underactuation, i.e., the inability to exert forces in some directions of the body frame. Quadrotors have been used as the main platform for applications as well as research, though they are also underactuated, i.e., they cannot exert any force parallel to the plane perpendicular to their vertical direction in body

\footnotetext{
${ }^{1}$ Max Planck Institute for Biological Cybernetics, Spemannstraße 38, 72076 Tübingen, Germany, \{sujit.rajappa\}\{hhb\}@tue.mpg.de

${ }^{2}$ Department of Brain and Cognitive Engineering, Korea University, Anam-dong, Seongbuk-gu, Seoul, 136-713 Korea.

${ }^{3}$ CNRS, LAAS, 7 avenue du colonel Roche, F-31400 Toulouse, France mryllelaas.fr, afranchi@laas.fr

${ }^{4}$ Univ de Toulouse, LAAS, F-31400 Toulouse, France

This work has been partially supported by the European Community under Contract ICT 287617 ARCAS.
}

frame. This is why a quadrotor needs to roll and pitch to accelerate in any direction different from a pure vertical one.

But when it comes to physical interaction, underactuation might become a serious problem for the capabilities and overall stabilization of the aerial vehicle. As the application complexity is going higher, major breakthroughs and advancements in innovative mechanical designs, actuation concepts, micro-electro mechanical systems, sensor technology and power capacity is always envisioned. Several possibilities have been proposed in the past literature spanning different concepts: ducted-fan designs [9], tilt-wing mechanisms [10], or tilt-rotor actuations [11], [12]. The concept of tilt-rotor architecture has been much explored to increase flight time [13] but not for the improvement of the underactuation problem. In [14] the underactuation was addressed by four additional rotors at the end of each frame in lateral position. But the position of the rotor increased the complexity of controllability because of the air flow between the vertical and the lateral rotors, resulting in non-linear dynamics along with the increase in payload.

Our own in-lab investigation led to the novel quadrotor design [15] with tilted propellers by 4 additional actuators included for the tilting thereby creating the possibility to regulate independently the 6 DoFs of the platform. Though underactuation problem was solved by this design, the need of tilting the propellers in order to resist to any external wrench makes it tough for the aerial manipulation task, where forces shall be exerted instantaneously to resist to unexpected external wrenches. Additionally, the use of servomotors for tilting the propellers makes the overall model challenging to control in real scenarios involving physical interaction.

In [16], a hexarotor with the propellers rotated by the same angle-magnitude about one axis was suggested. Our approach constitutes a generalization of [16], as we present a more general tilt design. Furthermore, w.r.t. [16] we present a new control law for 6 DoFs trajectory tracking, a methodology to optimize the fixed tilting angles for each propeller depending on the task in exam and an improved mechanical design where all the propellers lie in the same plane.

Taking inspiration from all the related work, we propose a novel hexarotor with tilted propeller design, where each rotor is fixedly mounted in a configuration that is rotated about two possible axes. The main objective of this work is full controllability of the UAV's position and orientation by means of tilted propellers, thereby making it completely actuated. The full actuation comes with the acceptable cost of a slightly more complex mechanical design. 


\begin{tabular}{|l|l|}
\hline Symbols & Definitions \\
\hline \hline$m$ & total mass of the hexarotor \\
\hline$g$ & gravity constant \\
\hline $\boldsymbol{O}_{B}$ & Center of hexarotor or Center of Mass (CoM) \\
\hline $\boldsymbol{O}_{P_{i}}$ & Center of the each propeller group \\
\hline $\boldsymbol{p}$ & position of $\boldsymbol{O}_{B}$ (the CoM) in $\mathcal{F}_{W}$ \\
\hline$\lambda_{i}$ & angular orientation of $\overline{\boldsymbol{O}}_{B}, \boldsymbol{O}_{P_{i}}$ on the $\boldsymbol{X}_{B} \boldsymbol{Y}_{B}$ plane \\
\hline$L_{x_{i}}$ & distance between $\boldsymbol{O}_{P_{i}}$ and $\boldsymbol{O}_{B}$ \\
\hline $\mathcal{F}_{W}$ & inertial world frame \\
\hline $\mathcal{F}_{B}$ & hexarotor body frame \\
\hline $\mathcal{F}_{P_{i}}$ & $i$-th propeller frame \\
\hline${ }^{W} \boldsymbol{R}_{B}$ & rotation matrix from $\mathcal{F}_{B}$ to $\mathcal{F}_{W}$ \\
\hline${ }^{B} \boldsymbol{R}_{P_{i}}$ & rotation matrix from $\mathcal{F}_{P_{i}}$ to $\mathcal{F}_{B}$ \\
\hline $\boldsymbol{I}_{B}$ & inertia of the hexarotor frame \\
\hline$k_{f}$ & propeller thrust coefficient \\
\hline$k_{m}$ & propeller drag coefficient \\
\hline$\alpha_{i}$ & $i$-th propeller tilt angle about $\overline{\boldsymbol{O}_{P_{i}} \boldsymbol{O}_{B}}$ \\
\hline$\beta_{i}$ & $i$-th propeller tilt angle about $\boldsymbol{Y}_{P_{i}}$ \\
\hline $\bar{\omega}_{i}$ & $i$-th propeller spinning velocity about $\boldsymbol{Z}_{P_{i}}$ \\
\hline $\boldsymbol{\omega}_{B}$ & angular velocity of $\mathcal{F}_{B}$ w.r.t. $\mathcal{F}_{W}$ expressed in $\mathcal{F}_{B}$ \\
\hline $\boldsymbol{\tau}_{\text {ext }}$ & external disturbance torque acting on the hexarotor \\
\hline $\boldsymbol{T}_{\text {thrust }}$ & $i$-th propeller thrust along $\boldsymbol{Z}_{P_{i}}$ \\
\hline $\boldsymbol{T}_{\text {drag }}$ & drag due to the $i$-th propeller along $\boldsymbol{Z}_{P_{i}}$ \\
\hline
\end{tabular}

TABLE I: Main symbols used in the paper

The focus and structure of this paper is therefore: $(i)$ to discuss in detail and derive the dynamic model for the proposed hexarotor in Sec. II, (ii) to devise and develop the closed-loop controller for the hexarotor which is able to asymptotically track an arbitrary desired trajectory for the position and orientation in 3-dimensional free space in Sec. III, ( iii) to optimize the propeller tilt angles depending on the application/trajectory to reduce the overall control effort in the Sec. IV-B, $(i v)$ to start the design of a novel and feasible hexarotor architecture with tilted propellers in Sec. IV, and finally $(v)$ to test the hexarotor model and its theoretical concepts in simulation, presented in Sec. V. Conclusions and future perspectives are given in Sec. VI.

\section{DESIGN AND MODELING}

A standard hexarotor possesses six propellers that are all rotating about six parallel axes. Even though this choice increases redundancy and payload, such configuration has an underactuated dynamics similar to a standard quadrotor. In fact, the six propellers create an input force that is always parallel to that axis, no matter the values of the six rotational speeds. In this case a change of the direction of the input force in world frame can only be obtained by reorienting the whole vehicle. As a consequence, the output trajectory can only be defined by a 4-dimensional output, namely the center of mass (CoM) 3D position plus the yaw angle, despite the presence of 6 control inputs. In fact in [17] it has been proven that such kind of systems are exactly linearizable with a dynamic feedback using as linearizing output, i.e. the CoM position and the yaw angle. Feedback linearizability also implies differential flatness of the system taking as flat output the linearizing one [18]. The remaining two configuration variables, i.e., the roll and pitch angles, cannot be chosen at will, since they are being determined by the desired trajectory of the CoM, the yaw angle, and their derivatives.

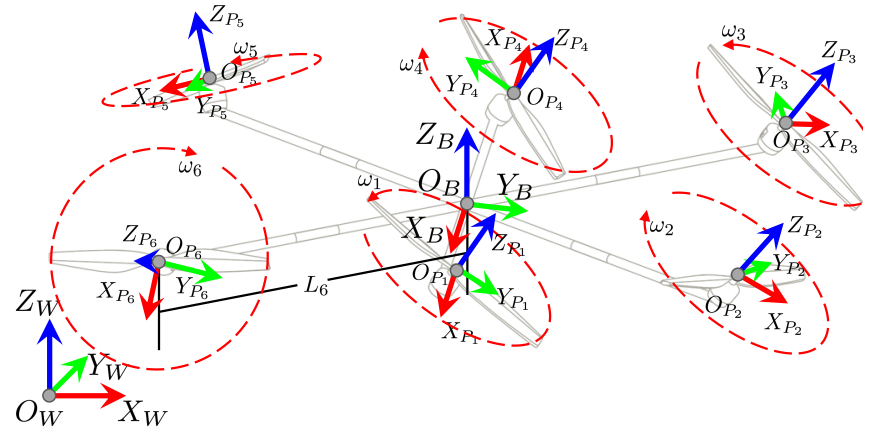

Fig. 1: Schematic representation of the hexarotor described in this paper.

On the converse, the goal of the hexarotor modeling approach presented here is to exploit at best the six available inputs, thus resulting in a system that is fully actuated, i.e., linear and angular accelerations can be set independently acting on the six inputs. In order to obtain full actuation, we remove the constraint for the propellers to rotate about six parallel axes, so that a force in any direction can be generated regardless of the vehicle orientation. Thanks to full actuation, this hexarotor can track 6-DoFs trajectories comprising both the CoM position and, independently, the vehicle orientation described, e.g., by roll, pitch, and yaw, or by a rotation matrix.

Even though a reallocation and reorientation of the six propellers allows for more design flexibility it also increases the number of design parameters thus increasing the design complexity. In order to find a good compromise between full actuation and low number of model parameters, we decide to add the following constraint on the parameters:

- the CoM and the six propeller centers are coplanar, like in a standard hexarotor;

This design choice simplify the design complexity while still allowing a full spectrum of actuation capabilities, as it will be shown in the paper.

The main symbols used in the paper are shown in Table I,

\section{A. Static System Description}

We denote the world inertial frame with $\mathcal{F}_{W}:\left\{\boldsymbol{O}_{W}-\right.$ $\left.\boldsymbol{X}_{W} \boldsymbol{Y}_{W} \boldsymbol{Z}_{W}\right\}$ and with $\mathcal{F}_{B}:\left\{\boldsymbol{O}_{B}-\boldsymbol{X}_{B} \boldsymbol{Y}_{B} \boldsymbol{Z}_{B}\right\}$ the body frame attached to the hexarotor frame, where $\boldsymbol{O}_{B}$ coincides with the hexarotor CoM. Let the frame associated with the $i$-th propeller be defined as $\mathcal{F}_{P_{i}}:\left\{\boldsymbol{O}_{P_{i}}-\boldsymbol{X}_{P_{i}} \boldsymbol{Y}_{P_{i}} \boldsymbol{Z}_{P_{i}}\right\}$, where $i=1 \ldots 6$. The origin $\boldsymbol{O}_{P_{i}}$ coincides with the center of spinning and the CoM of the $i$-th propeller, the axes $\boldsymbol{X}_{P_{i}}$ and $\boldsymbol{Y}_{P_{i}}$ define the rotation plane of the propeller, and $Z_{P_{i}}$ is the axis about which the propeller spins and coincides with the direction of the generated thrust force. The propeller frame $\mathcal{F}_{P_{i}}$ is rigidly attached to the hexarotor frame, rather than to the propeller, which spins about $Z_{P_{i}}$. In fact, only the direction of the force and torque exerted by the propeller are relevant to our problem. The actual spinning angle of each propeller is not important for the motion, as it will be explained in Sec. II-B.

We shall denote simply by $\boldsymbol{p} \in \mathbb{R}^{3}$ the position of $\boldsymbol{O}_{B}$ in $\mathcal{F}_{W}$, and by ${ }^{B} \boldsymbol{p}_{i} \in \mathbb{R}^{3}$ the position of $\boldsymbol{O}_{P_{i}}$ in $\mathcal{F}_{B}$, with 


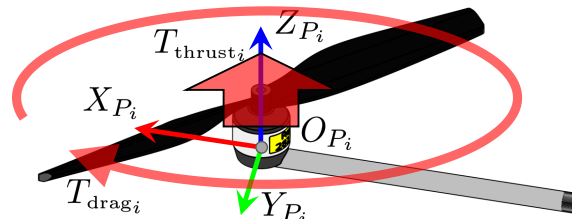

(a)

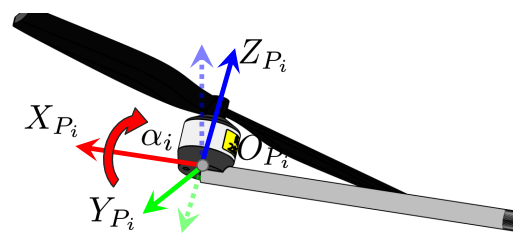

(b)

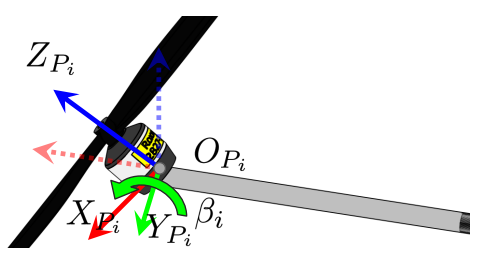

(c)

Fig. 2: (a): terminal part of the $i$-th hexarotor arm showing the body frame $\mathcal{F}_{P_{i}}$ and the generated thrust $T_{\text {thrust }}$ and drag $T_{\text {drag }_{i}}$; (b) and (c): Visualization of the possible reorientation of the propeller around $X_{P_{i}}$ (case (b)) and $Y_{P_{i}}$ (case (c)). The angle of reorientation is denoted with $\alpha_{i}$ in (b) and $\beta_{i}$ in (c)

$i=1 \ldots 6$. In order to have the 6 propellers centers lying on the $\boldsymbol{X}_{B} \boldsymbol{Y}_{B}$ plane we set:

$$
{ }^{B} \boldsymbol{p}_{i}=\boldsymbol{R}_{Z}\left(\lambda_{i}\right)\left[\begin{array}{c}
L_{x_{i}} \\
0 \\
0
\end{array}\right], \quad \forall i=1 \ldots 6
$$

where $\boldsymbol{R}_{Z}(\cdot)$ is the canonical rotation matrix about a $Z$-axis, $L_{x_{i}}>0$ is the distance between $\boldsymbol{O}_{P_{i}}$ and $\boldsymbol{O}_{B}$, and $\lambda_{i}$ is the angular direction of the segment $\overline{\boldsymbol{O}}_{B} \boldsymbol{O}_{P_{i}}$ on the $\boldsymbol{X}_{B} \boldsymbol{Y}_{B}$ plane.

The parameters $\lambda_{i}$ and $L_{x_{i}}$ should be chosen depending on the strength and length of propellers, size and shape of the hexarotor, payload needs, etc.. For example in Sec. V we shall choose $L_{x_{i}}=0.4 \mathrm{~m}$ and $\lambda_{i}=(i-1) \frac{\pi}{3}$.

Let the rotation matrix ${ }^{W} \boldsymbol{R}_{B} \in S O(3)$ represent the orientation of $\mathcal{F}_{B}$ w.r.t. $\mathcal{F}_{W}$ and ${ }^{B} \boldsymbol{R}_{P_{i}} \in S O(3)$ represent the orientation of $\mathcal{F}_{P_{i}}$ w.r.t. $\mathcal{F}_{B}$, for $i=1 \ldots 6$. In order to obtain a minimal parameterization of the propeller orientation we decompose each ${ }^{B} \boldsymbol{R}_{P_{i}}$ in three consecutive rotations

$$
{ }^{B} \boldsymbol{R}_{P_{i}}=\boldsymbol{R}_{Z}\left(\lambda_{i}\right) \boldsymbol{R}_{X}\left(\alpha_{i}\right) \boldsymbol{R}_{Y}\left(\beta_{i}\right), \quad \forall i=1 \ldots 6
$$

where the angular parameters $\alpha_{i}$ and $\beta_{i}$ represent the tilt angles that uniquely define the rotation plane of the $i$-th propeller, $\boldsymbol{X}_{P_{i}} \boldsymbol{Y}_{P_{i}}$ or, equivalently, the direction of $\boldsymbol{Z}_{P_{i}}$ in $\mathcal{F}_{B}$. The angles $\alpha_{i}$ and $\beta_{i}$ have a clear geometrical interpretation, in fact the $i$-th propeller plane $\boldsymbol{X}_{P_{i}} \boldsymbol{Y}_{P_{i}}$ is obtained from $\boldsymbol{X}_{B} \boldsymbol{Y}_{B}$ by first applying a rotation of $\alpha_{i}$ about the line $\overline{\boldsymbol{O}_{B} \boldsymbol{O}_{P_{i}}}$ and then a rotation of $\beta_{i}$ about $\boldsymbol{Y}_{P_{i}}$, which lies on $\boldsymbol{X}_{B} \boldsymbol{Y}_{B}$ and is perpendicular to $\overline{\boldsymbol{O}_{B} \boldsymbol{O}_{P_{i}}}$. The $\alpha_{i}$ and $\beta_{i}$ rotation is pictorially represented in Fig. 2.

For convenience, we group the following parameters into four 6-tuples: $\boldsymbol{\alpha}=\left(\alpha_{1}, \alpha_{2}, \alpha_{3}, \alpha_{4}, \alpha_{5}, \alpha_{6}\right), \boldsymbol{\beta}=$ $\left(\beta_{1}, \beta_{2}, \beta_{3}, \beta_{4}, \beta_{5}, \beta_{6}\right), \boldsymbol{\lambda}=\left(\lambda_{1}, \lambda_{2}, \lambda_{3}, \lambda_{4}, \lambda_{5}, \lambda_{6}\right)$ and $\boldsymbol{L}_{x}=\left(L_{x_{1}}, L_{x_{2}}, L_{x_{3}}, L_{x_{4}}, L_{x_{5}}, L_{x_{6}}\right)$.

In this paper we consider the case in which $\lambda_{i}, L_{x_{i}}, \alpha_{i}, \beta_{i}$, for $i=1 \ldots 6$, are constant during flight. Nevertheless, we allow $\alpha_{i}, \beta_{i}$ to be changed during a pre-flight setup, in order, e.g., to minimize the sum of the overall control effort for a specific task, as shown in Sec. IV-B.

\section{B. Equations of Motion}

Utilizing the standard Newton-Euler approach for dynamic systems, it is possible to derive the complete dynamic equations of the hexarotor by considering the forces and torques that are generated by each propeller rotation together with the significant gyroscopic and inertial effects. In the following we recap the standard ${ }^{1}$ assumptions that we are considering here:

- $\boldsymbol{O}_{B}$ coincides with the CoM of the hexarotor;

- $\boldsymbol{O}_{P_{i}}$ coincides with the CoM of the $i$-th propeller;

- the motors actuating the six propellers implement a fast high-gain local controller which is able to impose a desired spinning speed with negligible transient, thus allowing to consider the spinning rates as (virtual) control inputs in place of the motor torques;

- gyroscopic and inertial effects due to the propellers and the motors are considered as second-order disturbances to be rejected by the feedback nature of the controller;

- the tilted propellers might cause additional turbulences due to the possible intersection of the airflows. These turbulences are considered as negligible as the possible intersection of the airflows happens not close to the propellers. In fact, tilt configurations have been already proven to be feasible in reality [19].

We will test in simulation (see Section V) the practicability of these assumptions with the proposed controller on a dynamic model which includes the aforementioned unmodeled effects.

For ease of presentation, in the following we shall express the translational dynamics in $\mathcal{F}_{W}$ where as the rotational dynamics is expressed in $\mathcal{F}_{B}$.

1) Rotational dynamics: Denote with $\boldsymbol{\omega}_{B} \in \mathbb{R}^{3}$ the angular velocity of $\mathcal{F}_{B}$, with respect to $\mathcal{F}_{W}$, expressed in $\mathcal{F}_{B}$. Then the rotational dynamics is

$$
\boldsymbol{I}_{B} \dot{\boldsymbol{\omega}}_{B}=-\boldsymbol{\omega}_{B} \times \boldsymbol{I}_{B} \boldsymbol{\omega}_{B}+\boldsymbol{\tau}+\boldsymbol{\tau}_{\mathrm{ext}},
$$

where $\boldsymbol{I}_{B}$ is the hexarotor body inertia matrix, $\boldsymbol{\tau}_{\text {ext }}$ accounts for external disturbances and unmodeled effects, and $\tau$ is the input torque, which is decomposed in

$$
\tau=\tau_{\text {thrust }}+\tau_{\text {drag }},
$$

where $\tau_{\text {thrust }}$ is produced by the six propeller thrusts and $\tau_{\text {drag }}$ is due to the six propeller drags. The two individual components of (4) are discussed in detail below.

a) Torque due to thrusts $\left(\boldsymbol{\tau}_{\text {thrust }}\right)$ : The $i$-th propeller creates a force vector applied at $\boldsymbol{O}_{P_{i}}$ and directed along $\boldsymbol{Z}_{P_{i}}$, which is expressed in $\mathcal{F}_{P_{i}}$ by

$$
\boldsymbol{T}_{\text {thrust }_{i}}=\left[\begin{array}{lll}
0 & 0 & k_{f} \bar{\omega}_{i}^{2}
\end{array}\right]^{T}
$$

${ }^{1}$ Similar assumptions have been used, e.g., in [13]-[15] 
where $k_{f}>0$ is a constant thrust coefficient and $\bar{\omega}_{i}$ is the spinning velocity of the $i$-th propeller. The thrust torque, expressed in $\mathcal{F}_{B}$ is then

$$
\boldsymbol{\tau}_{\text {thrust }}=\sum_{i=1}^{6}\left({ }^{B} \boldsymbol{p}_{i} \times{ }^{B} \boldsymbol{R}_{P_{i}} \boldsymbol{T}_{\text {thrust }_{i}}\right) .
$$

b) Torque due to drag $\left(\tau_{\mathrm{drag}}\right)$ : The drag moment generated by the $i$-th propeller acts in the opposite direction of the propeller angular velocity and is expressed in $\mathcal{F}_{P_{i}}$ by

$$
\boldsymbol{T}_{\operatorname{drag}_{i}}=\left[\begin{array}{lll}
0 & 0 & (-1)^{i} k_{m} \bar{\omega}_{i}^{2}
\end{array}\right]^{T},
$$

where $k_{m}>0$ is the propeller drag coefficient. The factor $(-1)^{i}$ is used since half of the propellers rotate clockwise and the other half rotates counter-clockwise. This is done in order to have an automatic counterbalance of the drag torques at hovering. The drag torque due to the six propellers expressed in $\mathcal{F}_{B}$ is then

$$
\boldsymbol{\tau}_{\text {drag }}=\sum_{i=1}^{6}{ }^{B} \boldsymbol{R}_{P_{i}} \boldsymbol{T}_{\text {drag }_{i}} .
$$

Putting together (8) and (6) in (4) we can write

$$
\boldsymbol{\tau}=\boldsymbol{H}\left(\boldsymbol{\alpha}, \boldsymbol{\beta}, \boldsymbol{\lambda}, \boldsymbol{L}_{x}\right) \boldsymbol{u}
$$

where $\boldsymbol{H}\left(\boldsymbol{\alpha}, \boldsymbol{\beta}, \boldsymbol{\lambda}, \boldsymbol{L}_{x}\right) \in \mathbb{R}^{3 \times 6}$ is the matrix that relates the input torque $\tau$ to the control input

$$
\boldsymbol{u}=\left[\begin{array}{llllll}
\bar{\omega}_{1}^{2} & \bar{\omega}_{2}^{2} & \bar{\omega}_{3}^{2} & \bar{\omega}_{4}^{2} & \bar{\omega}_{5}^{2} & \bar{\omega}_{6}^{2}
\end{array}\right]^{T} \in \mathbb{R}^{6 \times 1},
$$

i.e., the squares of the rotational speeds of each propeller.

2) Translational dynamics: Thanks to the assumption on the location of the hexarotor and propeller centers of mass, we can express the translational dynamics in $\mathcal{F}_{W}$, using the standard Newton-Euler formulation, as

$$
m \ddot{\boldsymbol{p}}=m\left[\begin{array}{c}
0 \\
0 \\
-g
\end{array}\right]+{ }^{W} \boldsymbol{R}_{B} \boldsymbol{F}(\boldsymbol{\alpha}, \boldsymbol{\beta}, \boldsymbol{\lambda}) \boldsymbol{u}+\boldsymbol{f}_{\mathrm{ext}}
$$

where $\boldsymbol{f}_{\text {ext }}$ represents external disturbances and unmodeled effects, and $\boldsymbol{F}(\boldsymbol{\alpha}, \boldsymbol{\beta}, \boldsymbol{\lambda}) \in \mathbb{R}^{3 \times 6}$ is the matrix that relates $\boldsymbol{u}$ with the total force produced by the propellers (expressed in body frame), i.e.,

$$
\boldsymbol{F}(\boldsymbol{\alpha}, \boldsymbol{\beta}, \boldsymbol{\lambda}) \boldsymbol{u}=\sum_{i=1}^{6}{ }^{B} \boldsymbol{R}_{P_{i}} \boldsymbol{T}_{\text {thrust }_{i}} .
$$

Notice that in a standard hexarotor $\alpha_{i}=\beta_{i}=0$, for all $i=1 \ldots 6$. This implies that $\boldsymbol{F}(\boldsymbol{\alpha}, \boldsymbol{\beta}, \boldsymbol{\lambda})$ has rank equal to one (the total force is always directed on the $Z_{B}$ axis).

\section{CONTROL DESIGN}

The control problem considered here is an output tracking problem. In particular, the hexarotor is tasked to track, simultaneously, a desired trajectory $\boldsymbol{p}_{d}(t)$ with the CoM position $\boldsymbol{p}$ and a given orientation $\boldsymbol{R}_{d}(t)$ with the body orientation ${ }^{W} \boldsymbol{R}_{B}$. The available control inputs are the squares of the six spinning rates of the propellers $\boldsymbol{u}$ defined in (10).

Neglecting the external forces and torques (which are handled by the feedback nature of the control) we rewrite

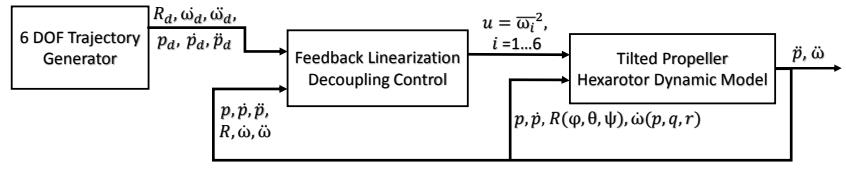

Fig. 3: Control scheme architecture

here the hexarotor dynamical model, that is used for the control design

$$
\begin{aligned}
\ddot{\boldsymbol{p}} & =\left[\begin{array}{lll}
0 & 0 & -g
\end{array}\right]^{T}+\frac{1}{m}{ }^{W} \boldsymbol{R}_{B} \boldsymbol{F}(\boldsymbol{\alpha}, \boldsymbol{\beta}, \boldsymbol{\lambda}) \boldsymbol{u} \\
\dot{\boldsymbol{\omega}}_{B} & =-\boldsymbol{I}_{B}^{-1}\left(\boldsymbol{\omega}_{B} \times \boldsymbol{I}_{B} \boldsymbol{\omega}_{B}\right)+\boldsymbol{I}_{B}^{-1} \boldsymbol{H}\left(\boldsymbol{\alpha}, \boldsymbol{\beta}, \boldsymbol{\lambda}, \boldsymbol{L}_{x}\right) \boldsymbol{u} \\
{ }^{W} \dot{\boldsymbol{R}}_{B} & ={ }^{W} \boldsymbol{R}_{B}\left[\boldsymbol{\omega}_{B}\right]_{\wedge}
\end{aligned}
$$

with $[\cdot]_{\wedge}$ being the hat operator from $\mathbb{R}^{3}$ to $s o(3)$.

\section{A. Exact Feedback Linearization and Decoupling Control}

In order to apply a feedback linearization technique we rewrite (13)-(14) in a matricial form

$\left[\begin{array}{c}\ddot{\boldsymbol{p}} \\ \dot{\boldsymbol{\omega}}_{B}\end{array}\right]=\boldsymbol{f}+\boldsymbol{J}_{R}\left[\overline{\boldsymbol{J}}\left(\boldsymbol{\alpha}, \boldsymbol{\beta}, \boldsymbol{\lambda}, \boldsymbol{L}_{x}\right)\right] \boldsymbol{u}=\boldsymbol{f}+\boldsymbol{J}\left(\boldsymbol{\alpha}, \boldsymbol{\beta}, \boldsymbol{\lambda}, \boldsymbol{L}_{x}\right) \boldsymbol{u}$

where $\boldsymbol{f} \in \mathbb{R}^{6}$ is the drift vector due to the gravity and the rotational inertia, $\boldsymbol{J}_{R}=\left[\begin{array}{cc}\frac{1}{m}{ }^{W} \boldsymbol{R}_{B} & \mathbf{0} \\ \mathbf{0} & \boldsymbol{I}_{B}^{-1}\end{array}\right] \in \mathbb{R}^{6 \times 6}$, $\overline{\boldsymbol{J}}\left(\boldsymbol{\alpha}, \boldsymbol{\beta}, \boldsymbol{\lambda}, \boldsymbol{L}_{x}\right)=\left[\begin{array}{c}\boldsymbol{F}(\boldsymbol{\alpha}, \boldsymbol{\beta}, \boldsymbol{\lambda}) \\ \boldsymbol{H}\left(\boldsymbol{\alpha}, \boldsymbol{\beta}, \boldsymbol{\lambda}, \boldsymbol{L}_{x}\right)\end{array}\right] \in \mathbb{R}^{6 \times 6}$. The $6 \times 6$ matrix $\boldsymbol{J}\left(\boldsymbol{\alpha}, \boldsymbol{\beta}, \boldsymbol{\lambda}, \boldsymbol{L}_{x}\right)$ is called the decoupling matrix ${ }^{2}$.

If $\boldsymbol{J}\left(\boldsymbol{\alpha}, \boldsymbol{\beta}, \boldsymbol{\lambda}, \boldsymbol{L}_{x}\right)$ is invertible we choose the control input as

$$
\boldsymbol{u}=\boldsymbol{J}^{-1}\left(\boldsymbol{\alpha}, \boldsymbol{\beta}, \boldsymbol{\lambda}, \boldsymbol{L}_{x}\right)(-\boldsymbol{f}+\boldsymbol{v})
$$

where $\boldsymbol{v}$ is an additional input, thus obtaining

$$
\left[\begin{array}{c}
\ddot{\boldsymbol{p}} \\
\dot{\boldsymbol{\omega}}_{B}
\end{array}\right]=\boldsymbol{v}=\left[\begin{array}{c}
\boldsymbol{v}_{p} \\
\boldsymbol{v}_{R}
\end{array}\right],
$$

i.e., the system is exactly linearized via a static feedback. Fig. 3 shows the control scheme architecture.

In order to obtain an exponential convergence to $\mathbf{0}$ of the position error $\boldsymbol{p}-\boldsymbol{p}_{d}=\boldsymbol{e}_{\boldsymbol{p}}$ one can choose a linear controller

$$
\boldsymbol{v}_{p}=\ddot{\boldsymbol{p}}_{d}-\boldsymbol{K}_{p 1} \dot{\boldsymbol{e}}_{\boldsymbol{p}}-\boldsymbol{K}_{p 2} \boldsymbol{e}_{\boldsymbol{p}}-\boldsymbol{K}_{p 3} \int_{t_{0}}^{t} \boldsymbol{e}_{\boldsymbol{p}}
$$

where the diagonal positive definite gain matrixes $\boldsymbol{K}_{p_{1}}, \boldsymbol{K}_{p_{2}}$, $\boldsymbol{K}_{p_{3}}$ define Hurwitz polynomials.

Now considering the orientation tracking, a popular used parameterization is to resort to Euler angles. However it is well known that they are prone to singularity problems. Keeping this in mind, the controller for the rotational configuration is developed directly on $S O(3)$ and thereby it avoids any singularities that arise in local coordinates, such as Euler

\footnotetext{
${ }^{2}$ In standard hexarotor the decoupling matrix $\boldsymbol{J}\left(\boldsymbol{\alpha}, \boldsymbol{\beta}, \boldsymbol{\lambda}, \boldsymbol{L}_{x}\right)$ has always rank equal to four, similarly to a quadrotor.
} 


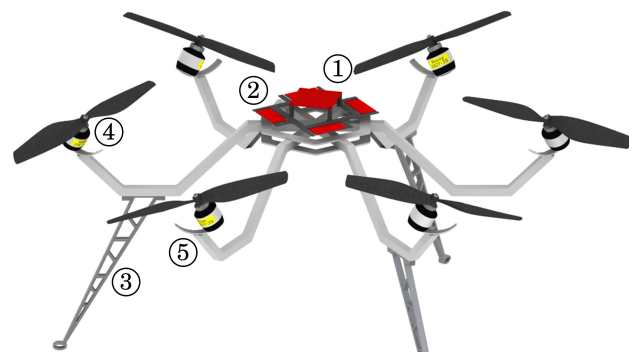

Fig. 4: CAD model of the preliminary prototype of the hexarotor with tilted propellers. It is composed of: (1) Micro controller, (2) Brushless controller, (3) Lander, (4) Propeller motor, (5) Tilting set-up.

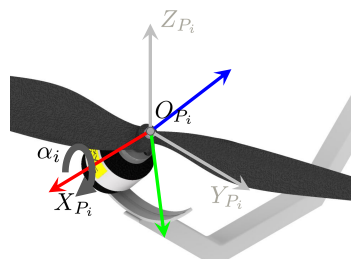

(a)

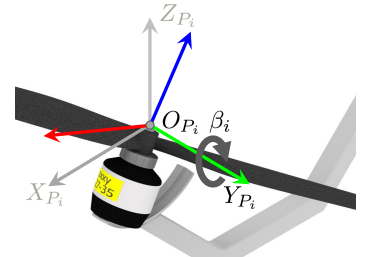

(b)
Fig. 5: (a) and (b): Visualization of the possible reorientation of the propeller around $X_{P_{i}}$ (case (a)) and $Y_{P_{i}}$ (case (b)). The angle of reorientation is denoted with $\alpha_{i}$ in (a) and $\beta_{i}$ in (b)

angles. Now assuming that $\boldsymbol{R}_{d}(t) \in \overline{\mathcal{C}}^{3}$ and $\boldsymbol{\omega}_{d}=\left[\boldsymbol{R}_{d}^{T} \dot{\boldsymbol{R}}_{d}\right]_{\vee}$, where $[\cdot]_{\vee}$ represents the inverse (vee) operator from so(3) to $\mathbb{R}^{3}$, the attitude tracking error $\boldsymbol{e}_{R} \in \mathbb{R}^{3}$ is defined similarly to $[20]$ as

$$
\boldsymbol{e}_{\boldsymbol{R}}=\frac{1}{2}\left[\boldsymbol{R}_{d}^{T W} \boldsymbol{R}_{B}-{ }^{W} \boldsymbol{R}_{B}^{T} \boldsymbol{R}_{d}\right]_{\vee},
$$

and the tracking error of the angular velocity $\boldsymbol{e}_{\omega} \in \mathbb{R}^{3}$ is given by

$$
\boldsymbol{e}_{\boldsymbol{\omega}}=\boldsymbol{\omega}_{B}-{ }^{W} \boldsymbol{R}_{B}^{T} \boldsymbol{R}_{d} \boldsymbol{\omega}_{d}
$$

In order to obtain an asymptotic convergence to $\mathbf{0}$ of the rotational error $e_{R}$ one can choose the following controller

$$
\boldsymbol{v}_{R}=\dot{\omega}_{d}-\boldsymbol{K}_{R_{1}} \boldsymbol{e}_{\omega}-\boldsymbol{K}_{R_{2}} \boldsymbol{e}_{R}-\boldsymbol{K}_{R_{3}} \int_{t_{0}}^{t} \boldsymbol{e}_{R}
$$

where the diagonal positive definite gain matrixes $\boldsymbol{K}_{R_{1}}$, $\boldsymbol{K}_{R_{2}}, \boldsymbol{K}_{R_{3}}$ define Hurwitz polynomials also in this case.

\section{A PRELIMINARY PROTOTYPE}

In this section we present the design of a preliminary prototype obtained instantiating the general model introduced in Section II in a more particular case. A CAD of the prototype is shown in Fig. 4.

First of all, to reduce the complexity and for the sake of symmetry, we have chosen $\lambda_{i}=(i-1) \frac{\pi}{3}$ and $L_{x_{i}}=$ $0.4 \mathrm{~m} \forall i=1 \ldots 6$. With this choice, the origin $\boldsymbol{O}_{P_{i}}$ of each propeller frame is equally spaced with $60^{\circ}$ between each other from the center of the body frame $\boldsymbol{O}_{B}$ to have a symmetric configuration in normal hovering position.

Furthermore, as shown in Fig. 5 each propeller is mounted in an arc frame which is free to rotate in $\boldsymbol{X}_{P_{i}}$ and $\boldsymbol{Y}_{P_{i}}$, so

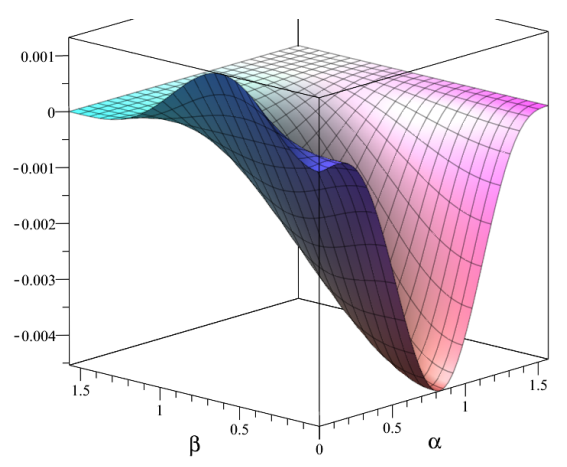

Fig. 6: Determinant value (z-axis) of $\overline{\boldsymbol{J}}(\boldsymbol{\alpha}, \boldsymbol{\beta})$ of the presented prototype

that the tilt angle of $\alpha_{i}$ and $\beta_{i}$ can be fixed as desired. The radius of the $\operatorname{arc}\left(R_{a r c}\right)$ is designed equal to the length of the motor (with the propeller attached), so that $\boldsymbol{O}_{P_{i}}$ always stays at the same location in the $\boldsymbol{X}_{B} \boldsymbol{Y}_{B}$ plane with only its direction vector $\left[\begin{array}{lll}\boldsymbol{X}_{P_{i}} & \boldsymbol{Y}_{P_{i}} & \boldsymbol{Z}_{P_{i}}\end{array}\right]^{T}$ changing according to the $\alpha_{i}$ and $\beta_{i}$ orientation.

The arm in which each propeller set-up is suspended is designed to have a curved architecture with the radius of the curvature, more than the propeller radius $\left(R_{\text {prop }}\right)$, so that independently from the value of $\alpha_{i}$ and $\beta_{i}$ in a certain allowed interval, the propellers never come in contact with the arm during flight.

Finally in this preliminary prototype we consider the following constraints

$$
\begin{aligned}
& \alpha_{1}= \pm \alpha_{2}= \pm \alpha_{3}= \pm \alpha_{4}= \pm \alpha_{5}= \pm \alpha_{6}=\alpha \\
& \beta_{1}= \pm \beta_{2}= \pm \beta_{3}= \pm \beta_{4}=\beta_{5}= \pm \beta_{6}=\beta .
\end{aligned}
$$

\section{A. Discussion on the Invertibility of $\boldsymbol{J}(\alpha, \beta)$}

In this prototype the decoupling matrix depends only on the choice of $\alpha$ and $\beta$. Our control algorithm relies on the invertibility of $\boldsymbol{J}(\alpha, \beta)$. This implies $\rho_{J}=\operatorname{rank}(\boldsymbol{J}(\alpha, \beta))=$ $\operatorname{rank}\left(\boldsymbol{J}_{R} \overline{\boldsymbol{J}}(\alpha, \beta)\right) \equiv \operatorname{rank}(\overline{\boldsymbol{J}}(\alpha, \beta))=6, \forall t>0$. Here $\boldsymbol{J}_{R}$ is a nonsingular square matrix as seen in (16) and therefore does not affect $\operatorname{rank}(\boldsymbol{J}(\alpha, \beta))$. Therefore $\overline{\boldsymbol{J}}(\alpha, \beta)$ is the only rank affecting component.

Due to the high non linearity of $\overline{\boldsymbol{J}}(\alpha, \beta)$ sufficient conditions for the invertibility are hard to find. Just to give an example, Fig. 6 shows $\operatorname{det}(\overline{\boldsymbol{J}}(\alpha, \beta))$ for a particular choice of the pluses and minuses in (23) and (24).

\section{B. Optimization of $\alpha$ and $\beta$}

The angles $\alpha$ and $\beta$ can be adjusted during the preflight setup. This gives the possibility to change the angles depending on the needs of a particular trajectory. In this section, we consider this capability to optimize $\alpha$ and $\beta$ depending on a predefined desired trajectory to reduce the control effort. As a reminder, the main motive is the full controllability in position and orientation. This comes with the cost of a higher control effort. The objective of this section is therefore to reduce this parasitic effect.

The predominant energy consuming parts of the hexarotor are the propeller motors. Minimizing the control effort 


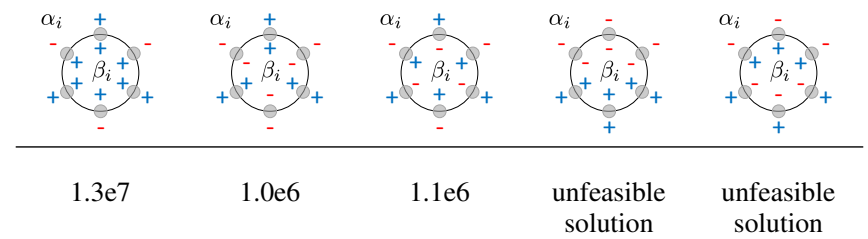

(a)

(b)

(c)

(d)

(e)

TABLE II: Stylized tested configuration and results. First row: Different configuration presented. Outside the circle the sign of $\alpha_{i}$ is indicated, within the circle the sign of $\beta_{i}$ is indicated. Second row: Value of the optimized $\int\|\boldsymbol{u}\|_{\min }$. Configuration (b) is the best configuration for the given trajectory. Configurations (d) and (e) are not feasible configurations

through the norm of the control output $\|\boldsymbol{u}\|$ by optimizing the particular $\alpha$ and $\beta$ will as well reduce the energy consumption in flight.

To reduce the complexity of the optimization, $\alpha_{i}$ and $\beta_{i}$ shall be changed in a coordinated way as explained before. We decided to use the same $\alpha$ and $\beta$ respectively for $\alpha_{i}$ and $\beta_{i} \in i=1 \ldots 6$, but with different signs for the individual joints (see (23) and (24)). An overview of the compared configurations can be found in table II.

The coordinated variation of $\alpha_{i}$ and $\beta_{i}$ offers two additional advantages: $(i)$ no asymmetries in the hexarotor body and $(i i)$ none or a minimum change of the CoM.

Considering these constraints, the optimization problem can be defined as:

$$
\min _{\alpha, \beta} \int_{0}^{t_{f}}\|\boldsymbol{u}\| \mathrm{d} t
$$

Subject to:

$$
\begin{aligned}
& 0<\alpha<\frac{\pi}{2} \\
& 0<\beta<\frac{\pi}{2} \\
& 0<\bar{\omega}_{i}, \text { for } i \in 1 . .6
\end{aligned}
$$

Here (26) and (27) are defining the lower and upper bounds for $\alpha_{i}$ and $\beta_{i}$, while (28) ensures a positive rotation speed $\bar{\omega}_{i}$ for all propellers. The presented minimization problem is a multi-dimensional constrained nonlinear optimization problem and can be solved using the in-build optimization capabilities of MATLAB by exploiting the fmincon-function [21].

To compare (minimal control effort) the different configurations shown in table II, we used the presented optimization technique to find the optimal values $\alpha^{\star}$ and $\beta^{\star}$ and the associated $\int\|\omega\|_{\text {min }}$. As trajectory, a typical flight regime has been chosen, which is presented in Section V-A. The minimum value of the objective function could be found in configuration (b). Therefore all further experiments will be performed by using this configuration: $\alpha=\alpha_{1}=-\alpha_{2}=$ $\alpha_{3}=-\alpha_{4}=\alpha_{5}=-\alpha_{6}$ and $\beta=\beta_{1}=-\beta_{2}=\beta_{3}=-\beta_{4}=$ $\beta_{5}=-\beta_{6}$.

The optimal angles $\alpha^{\star}$ and $\beta^{\star}$ are highly dependent on the desired trajectory. To visualize the influence we conducted a trajectory, where the hexarotor hovers in place but performs

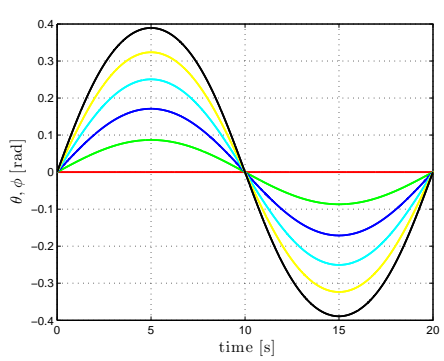

(a)

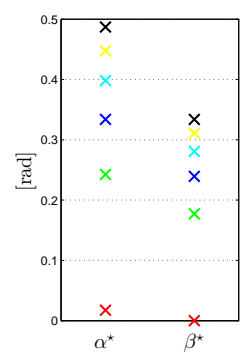

(b)
Fig. 7: (a): Desired sinusoidal trajectories for equal $\theta$ and $\phi$. Their amplitude is increased in six steps from $0^{\circ}(0 \mathrm{rad})$ to $22.5^{\circ}(\approx$ $0.39 \mathrm{rad})$; all other values remain constant $(=0)$. (b): Optimal values for $\alpha$ and $\beta$ corresponding to the six trajectories presented in (a)

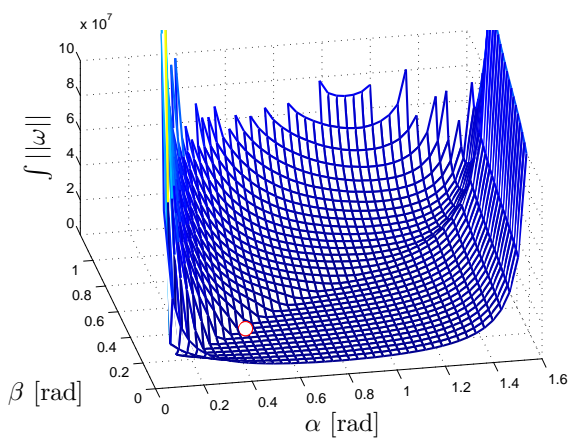

Fig. 8: Objective function for a given trajectory. Optimum is marked with a red circle

a sinusoidal rotation around $\theta$ and $\phi$ at the same time (see figure 7 (a)). The magnitude of the rotation is increased in 6 steps up to $22.5^{\circ} . \alpha^{\star}$ and $\beta^{\star}$ are increasing accordingly from almost zero values to $\alpha^{\star}=0.49 \mathrm{rad}$ and $\beta^{\star}=0.33 \mathrm{rad}$ for the maximum amplitude.

Figure 8 shows the influence of the optimization itself. For the considered sinusoidal trajectory, we calculated the value of the objective function for a wide variety of $\alpha^{\star}$ and $\beta^{\star}$. The optimal value is marked by a red circle in Fig. 8.

\section{SIMULATIONS AND RESULTS}

Here, we intend to present two simulations performed on the novel tilted propeller hexarotor. We aim to prove two important features of the proposed platform: (i) the ability of reorienting while hovering and reacting to external force/torque disturbances, (ii) 6-DoF (position+orientation) trajectory tracking. Given the chosen $\boldsymbol{\alpha}$ and $\boldsymbol{\beta}$, not all trajectories might be feasible since the negative control outputs $u_{i}$ might occur. This needs to be considered during the pre-trajectory planning step.

\section{A. Reorienting while hovering with external disturbance}

In the first simulation, we tested a hovering trajectory in which the hexarotor maintains a fixed position $\boldsymbol{p}$ but reorients itself changing at the same time the roll $\phi$, pitch $\theta$ and yaw $\psi$ angles. This involves hexarotor orienting $-12^{\circ}$ w.r.t. $\boldsymbol{X}_{B}, 12^{\circ}$ w.r.t. $\boldsymbol{Y}_{B}$ axis and $15^{\circ}$ w.r.t. $\boldsymbol{Z}_{B}$ while still hovering in the position $\boldsymbol{p}=\left[\begin{array}{lll}0 & 0 & 0\end{array}\right]^{T}$. Notice that orienting 


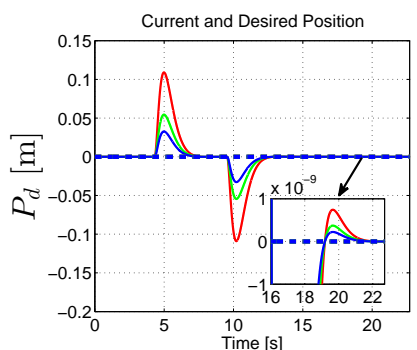

(a)



(c)

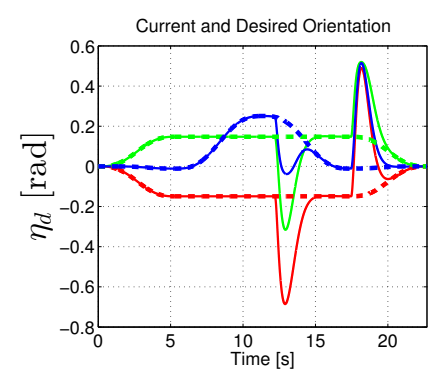

(b)

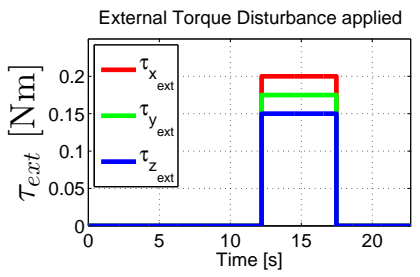

(d)
Fig. 9: Results of the hovering with external force/torque disturbance. 9(a): Desired (dashed line) and current (solid line) position $\boldsymbol{p}_{d}$ in $\mathrm{x}(\mathrm{red}), \mathrm{y}$ (green) and $\mathrm{z}(\mathrm{blue}) .9(\mathrm{~b})$ : Desired (dashed line) and current (solid line) orientation $\boldsymbol{\eta}_{d}$ in roll(red), pitch(green) and yaw(blue). 9(c-d): external force $\left(\boldsymbol{f}_{\text {ext }}\right)$ and torque $\left(\boldsymbol{\tau}_{\text {ext }}\right)$ applied to the hexarotor

w.r.t. the 3 principal body axes $\left\{\boldsymbol{X}_{B}, \boldsymbol{Y}_{B}, \boldsymbol{Z}_{B}\right\}$ while holding the same position is not feasible in a standard (coplanar) hexarotor UAV. The initial conditions were set to $\boldsymbol{p}\left(t_{0}\right)=\mathbf{0}, \dot{\boldsymbol{p}}\left(t_{0}\right)=\mathbf{0},{ }^{W} \boldsymbol{R}_{B}\left(t_{0}\right)=\mathbf{I}_{3}$ and $\boldsymbol{\omega}_{B}\left(t_{0}\right)=$ $\mathbf{0}$. The desired trajectory was chosen as $\boldsymbol{p}_{d}(t)=0$ and $\boldsymbol{R}_{d}(t)=\boldsymbol{R}_{X}(\phi(t)) \boldsymbol{R}_{Y}(\theta(t)) \boldsymbol{R}_{Z}(\psi(t))$ with $\phi(t), \theta(t), \psi(t)$ following a smooth profile having as maximum velocity $\dot{\theta}_{\max }=5^{\circ} / \mathrm{s}$ and maximum acceleration $\ddot{\theta}_{\max }=2.5^{\circ} / \mathrm{s}^{2}$. The optimized value of $\alpha^{\prime}=13.6^{\circ}$ and $\beta^{\prime}=10.6^{\circ}$ obtained from Sec. IV-B has been used. The gains in Equations (19) and (22) were set to $\boldsymbol{K}_{p_{1}}=\boldsymbol{K}_{R_{1}}=10 \mathbf{I}_{3}, \boldsymbol{K}_{p_{2}}=\boldsymbol{K}_{R_{2}}=$ $29 \mathbf{I}_{3}$ and $\boldsymbol{K}_{p_{3}}=\boldsymbol{K}_{R_{3}}=30 \mathbf{I}_{3}$.

Figures $9(a-d)$ show the result of hovering with external force/torque disturbance. As clearly seen in Fig. 9(c) a constant external force disturbance $\left(\boldsymbol{f}_{\text {ext }}=\left[\begin{array}{lll}4 & 2 & 1\end{array}\right]^{T} \mathrm{~N}\right)$ is applied, along the 3 principal axis $\left\{\boldsymbol{X}_{B}, \boldsymbol{Y}_{B}, \boldsymbol{Z}_{B}\right\}$, from $t=4$ to $9 \mathrm{~s}$. Fig. 9(a) shows the position (current (solid line) and desired (dashed line)) brought under control while $f_{\text {ext }}$ is applied thanks to the integral term in (19). Similarly in Fig. 9(d) a constant external torque disturbance $\left(\boldsymbol{\tau}_{\mathrm{ext}}=\right.$ $\left.\left[\begin{array}{lll}0.2 & 0.175 & 0.15\end{array}\right]^{T} \mathrm{Nm}\right)$ is applied, about the 3 principal axes $\left\{\boldsymbol{X}_{B}, \boldsymbol{Y}_{B}, \boldsymbol{Z}_{B}\right\}$, from $t=12$ to 18 s. Fig. 9(b) shows the orientation that gets disturbed by this external torque and brought under control after a short transient, thanks to the integral term in (22). The in-zoomed Fig. 9(a) shows that the position tracking error is very minimal in powers of $10^{-9}$. This simulation provides a first confirmation of the validity of the robustness of the controller during hovering with external disturbance and also the ability of reorienting the hexarotor while maintaining a fixed position, thus showing the 6-DoF capabilities of the hexarotor. This point will also be addressed more thoroughly by the next simulation.

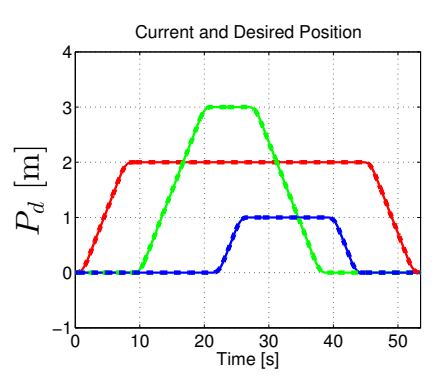

(a)

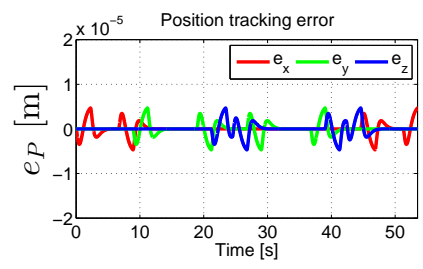

(c)

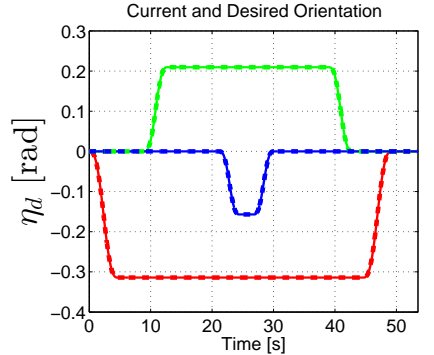

(b)

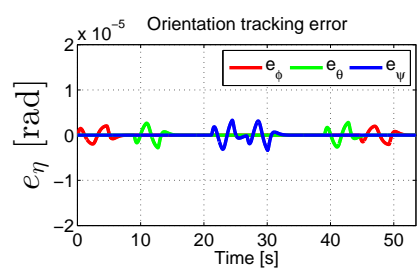

(d)
Fig. 10: Results of the robust 6 DoFs trajectory tracking. 10(a): Desired (dashed line) and current (solid line) position $\boldsymbol{p}_{d}$ in x(red), $\mathrm{y}($ green) and $\mathrm{z}$ (blue). 10(b): Desired (dashed line) and current (solid line) orientation $\boldsymbol{\eta}_{d}$ in roll(red), pitch(green) and yaw(blue). 10(cd): behavior of the position/orientation tracking errors $\left(\boldsymbol{e}_{p}, \boldsymbol{e}_{\eta}\right)$.

\section{B. 6 DoF trajectory tracking}

In this simulation, we have addressed a more complex trajectory following a square path with vertexes $\left\{V_{1}, V_{2}, V_{3}, V_{4}, V_{5}, V_{6}, V_{7}\right\}$. Each vertex was associated with the following desired positions and orientations ${ }^{3}$

- $\boldsymbol{V}_{1}: \boldsymbol{p}_{d}=\left[\begin{array}{lll}0 & 0 & 0\end{array}\right]^{T}, \boldsymbol{\eta}_{d}=\left[\begin{array}{lll}0^{\circ} & 0^{\circ} & 0^{\circ}\end{array}\right]^{T}$

- $\boldsymbol{V}_{2}: \boldsymbol{p}_{d}=\left[\begin{array}{lll}2 & 0 & 0\end{array}\right]^{T}, \boldsymbol{\eta}_{d}=\left[\begin{array}{lll}-18^{\circ} & 0^{\circ} & 0^{\circ}\end{array}\right]^{T}$

- $\boldsymbol{V}_{3}: \boldsymbol{p}_{d}=\left[\begin{array}{lll}2 & 3 & 0\end{array}\right]^{T}, \boldsymbol{\eta}_{d}=\left[\begin{array}{lll}-18^{\circ} & 12^{\circ} & 0^{\circ}\end{array}\right]^{T}$

- $\boldsymbol{V}_{4}: \boldsymbol{p}_{d}=\left[\begin{array}{lll}2 & 3 & 1\end{array}\right]^{T}, \boldsymbol{\eta}_{d}=\left[\begin{array}{lll}-18^{\circ} & 12^{\circ} & 9^{\circ}\end{array}\right]^{T}$

- $\boldsymbol{V}_{5}: \boldsymbol{p}_{d}=\left[\begin{array}{lll}2 & 0 & 1\end{array}\right]^{T}, \boldsymbol{\eta}_{d}=\left[\begin{array}{lll}-18^{\circ} & 12^{\circ} & 0^{\circ}\end{array}\right]^{T}$

- $\boldsymbol{V}_{6}: \boldsymbol{p}_{d}=\left[\begin{array}{lll}2 & 0 & 0\end{array}\right]^{T}, \boldsymbol{\eta}_{d}=\left[\begin{array}{lll}-18^{\circ} & 0^{\circ} & 0^{\circ}\end{array}\right]^{T}$

- $\boldsymbol{V}_{7}: \boldsymbol{p}_{d}=\left[\begin{array}{lll}0 & 0 & 0\end{array}\right]^{T}, \boldsymbol{\eta}_{d}=\left[\begin{array}{lll}0^{\circ} & 0^{\circ} & 0^{\circ}\end{array}\right]^{T}$

which were traveled along with rest-to-rest motions with maximum linear/angular velocities of $0.3 \mathrm{~m} / \mathrm{s}$ and $15^{\circ} / \mathrm{s}$, and maximum linear/angular accelerations of $0.2 \mathrm{~m} / \mathrm{s}^{2}$ and $5^{\circ} / \mathrm{s}$. Figures 10(a-d) show the desired trajectory $\left(\boldsymbol{p}_{d}(t), \boldsymbol{\eta}_{d}(t)\right)$, and the tracking errors $\left(\boldsymbol{e}_{p}(t), \boldsymbol{e}_{R}(t)\right)$. The same initial condition as in Sec. V-A is considered. The optimized value of $\alpha^{\prime}=26.5^{\circ}$ and $\beta^{\prime}=19^{\circ}$ for this trajectory obtained from Sec. IV-B has been used. Here it is clearly illustrated that at the vertex $\boldsymbol{V}_{4}$ the hexarotor exploits the 6 DoFs. Note again how the tracking errors are kept to minimum (in power of $10^{-5}$ ) despite the more complex motion involving several reorientations of the propellers. This confirms again the validity of the proposed controller.

The interested reader can refer to the video attached to the paper for a more exhaustive illustration of the hovering and 6-DoF hexarotor motion capabilities.

\footnotetext{
${ }^{3}$ Here, for the sake of clarity, we represent orientations by means of the classical roll/pitch/yaw Euler set $\boldsymbol{\eta} \in \mathbb{R}^{3}$.
} 


\section{CONCLUSIONS AND FUTURE WORKS}

In this paper, we have overcome the underactuation issues of a standard UAV with a tilted propeller hexarotor architecture where the propellers can be rotated both w.r.t. X-axis and Y-axis. This concept allows to $(i)$ gain full controllability over the 6 DoFs hexarotor pose in free space, and (ii) optimize the propeller tilt angle with respect to a minimum control effort over a desired trajectory. Carefully analyzing the controllability properties of the dynamic model, resulted in the hexarotor design being combined with trajectory tracking controller based on feedback linearization techniques. A clear validation of the controller's robustness is proved by means of extensive simulations.

Our future works are aimed at $(i)$ realizing the prototype of the proposed tilted propeller hexarotor in order to experimentally validate the ideas discussed in this paper. Further research is mandatory for feasibility of $\boldsymbol{u}$. In addition, we are also, $(i i)$ proceeding in the direction of optimizing the tilt angle to get a desired force $\left(\boldsymbol{F}_{x}, \boldsymbol{F}_{y}, \boldsymbol{F}_{z} \in \mathbb{R}^{3}\right)$ and desired torque $\left(\boldsymbol{T}_{x}, \boldsymbol{T}_{y}, \boldsymbol{T}_{z} \in \mathbb{R}^{3}\right)$ along the 3 principal body axes $\left\{\boldsymbol{X}_{B}, \boldsymbol{Y}_{B}, \boldsymbol{Z}_{B}\right\}$, which is a major objective for the aerial physical interaction with the environment.

\section{REFERENCES}

[1] A. Franchi, C. Secchi, M. Ryll, H. H. Bülthoff, and P. Robuffo Giordano, "Shared control: Balancing autonomy and human assistance with a group of quadrotor UAVs," IEEE Robotics \& Automation Magazine, Special Issue on Aerial Robotics and the Quadrotor Platform, vol. 19, no. 3, pp. 57-68, 2012.

[2] L. Marconi, R. Naldi, and L. Gentili, "Modeling and control of a flying robot interacting with the environment," Automatica, vol. 47, no. 12, pp. 2571-2583, 2011.

[3] G. Gioioso, M. Ryll, D. Prattichizzo, H. H. Bülthoff, and A. Franchi, "Turning a near-hovering controlled quadrotor into a 3D force effector," in 2014 IEEE Int. Conf. on Robotics and Automation, Hong Kong, China, May. 2014, pp. 6278-6284.

[4] B. Yüksel, C. Secchi, H. H. Bülthoff, and A. Franchi, "Reshaping the physical properties of a quadrotor through IDA-PBC and its application to aerial physical interaction," in 2014 IEEE Int. Conf. on Robotics and Automation, Hong Kong, China, May. 2014, pp. 62586265.

[5] — "A nonlinear force observer for quadrotors and application to physical interactive tasks," in 2014 IEEE/ASME Int. Conf. on Advanced Intelligent Mechatronics, Besançon, France, Jul. 2014, pp. 433-440.

[6] P. E. I. Pounds, D. R. Bersak, and A. M. Dollar, "Grasping from the air: Hovering capture and load stability," in 2011 IEEE Int. Conf. on Robotics and Automation, Shanghai, China, May 2011, pp. 24912498.
[7] Q. Lindsey, D. Mellinger, and V. Kumar, "Construction of cubic structures with quadrotor teams," in 2011 Robotics: Science and Systems, Los Angeles, CA, Jun. 2011.

[8] G. Gioioso, A. Franchi, G. Salvietti, S. Scheggi, and D. Prattichizzo, "The Flying Hand: a formation of uavs for cooperative aerial telemanipulation," in 2014 IEEE Int. Conf. on Robotics and Automation, Hong Kong, China, May. 2014, pp. 4335-4341.

[9] R. Naldi, L. Gentili, L. Marconi, and A. Sala, "Design and experimental validation of a nonlinear control law for a ducted-fan miniature aerial vehicle," Control Engineering Practice, vol. 18, no. 7, pp. 747760, 2010.

[10] K. T. Oner, E. Cetinsoy, M. Unel, M. F. Aksit, I. Kandemir, and K. Gulez, "Dynamic model and control of a new quadrotor unmanned aerial vehicle with tilt-wing mechanism," Int. Journ. of Mechanical, Aerospace, Industrial and Mechatronics Engineering, vol. 2, no. 9, pp. 12-17, 2008.

[11] F. Kendoul, I. Fantoni, and R. Lozano, "Modeling and control of a small autonomous aircraft having two tilting rotors," IEEE Trans. on Robotics, vol. 22, no. 6, pp. 1297-1302, 2006.

[12] A. Sanchez, J. Escareño, O. Garcia, and R. Lozano, "Autonomous hovering of a noncyclic tiltrotor UAV: Modeling, control and implementation," in 17th IFAC World Congress, Seoul, South Korea, Jul 2008, pp. 803-808.

[13] G. R. Flores, J. E. no, R. Lozano, and S. Salazar, "Quad-tilting rotor convertible MAV: Modeling and real-time hover flight control," Journal of Intelligent \& Robotics Systems, vol. 65, no. 1-4, pp. 457471, 2011.

[14] S. Salazar, H. Romero, R. Lozano, and P. Castillo, "Modeling and real-time stabilization of an aircraft having eight rotors," Journal of Intelligent \& Robotics Systems, vol. 54, no. 1-3, pp. 455-470, 2008.

[15] M. Ryll, H. H. Bülthoff, and P. Robuffo Giordano, "Modeling and control of a quadrotor UAV with tilting propellers," in 2012 IEEE Int. Conf. on Robotics and Automation, St. Paul, MN, May 2012, pp. 4606-4613.

[16] R. Voyles and G. Jiang, "Hexrotor UAV platform enabling dextrous interaction with structurespreliminary work," in 2012 IEEE Int. Symp. on Safety, Security and Rescue Robotics, College Station, TX, Nov. 2012, pp. 1-7.

[17] V. Mistler, A. Benallegue, and N. K. M'Sirdi, "Exact linearization and noninteracting control of a 4 rotors helicopter via dynamic feedback," in 10th IEEE Int. Symp. on Robots and Human Interactive Communications, Bordeaux, Paris, France, Sep. 2001, pp. 586-593.

[18] A. De Luca and G. Oriolo, "Trajectory planning and control for planar robots with passive last joint," The International Journal of Robotics Research, vol. 21, no. 5-6, pp. 575-590, 2002.

[19] M. Ryll, H. H. Bülthoff, and P. Robuffo Giordano, "First flight tests for a quadrotor UAV with tilting propellers," in 2013 IEEE Int. Conf. on Robotics and Automation, Karlsruhe, Germany, May 2013, pp. 295302.

[20] T. Lee, M. Leoky, and N. H. McClamroch, "Geometric tracking control of a quadrotor UAV on SE(3)," in 49th IEEE Conf. on Decision and Control, Atlanta, GA, Dec. 2010, pp. 5420-5425.

[21] Y. Cheon, D. Lee, I.-B. Lee, and S. W. Sung, "A new PID autotuning strategy with operational optimization for MCFC systems," in 9th Asian Control Conference, Istanbul, Turkey, Jun. 2013, pp. 1-6. 